\title{
The VEGF- and PDGF-family of angiogenic markers have prognostic impact in soft tissue sarcomas arising in the extremities and trunk
}

Thomas K Kilvaer ${ }^{1 *}$, Eivind Smeland ${ }^{1}$, Andrej Valkov²,3, Sveinung W Sorbye ${ }^{2,3}$, Roy M Bremnes ${ }^{1,4}$, Lill-Tove Busund ${ }^{2,3}$ and Tom Donnem ${ }^{1,4}$

\begin{abstract}
Background: Soft-tissue sarcomas are rare malignant tumors of mesenchymal lineage that can arise in any part of the body. Prognosis, and hence also treatment may vary according to histologic subtype and localization.

Angiogenesis is the process of forming new blood vessels from pre-existing ones. The deregulation of this process is thought to be an important step in malignant transformation. This study investigates the prognostic impact of platelet derived growth factor- (PDGF), vascular endothelial growth factor- (VEGF) and fibroblast growth factor (FGF) families in soft-tissue sarcomas of the extremities \& trunk (ET) and visceral \& retroperitoneal (VR) locations.

Methods: Tumor samples from 181 patients (115 ET and 66 VR) with resected soft tissue sarcomas were collected and tissue microarrays were constructed. Immunohistochemistry was used to evaluate angiogenic marker expression. Recurrence-free survival (RFS), metastasis-free survival (MFS) and disease-specific survival (DSS) were used as endpoints in prognostic impact assessment.

Results: In univariate analyses, almost all investigated angiogenic markers had prognostic impact in the ET group. In contrast, only FGFR-1 showed any significant prognostic impact in the VR group. In the multivariate analyses, PDGF-D $(H R=1.863,95 \% \mathrm{Cl}=1.057-3.283, \mathrm{P}=0.031)$, VEGFR-1 $(\mathrm{HR}=2.106,95 \% \mathrm{Cl}=1.038-4.272, \mathrm{P}=0.039)$ and VEGF-A (HR 2.095, 95\% Cl 1.028-4.271, $\mathrm{P}=0.042$ ) were independent negative prognosticators for DSS, MFS and RFS, respectively, in the ET group. FGFR-1 was an independent positive prognosticator for DSS ( $H R=0.243,95 \% \mathrm{Cl}=0.095-0.618, \mathrm{P}=0.003)$ in the VR group.
\end{abstract}

Conclusions: Angiogenic molecules from the PDGF and VEGF families have prognostic impact in soft-tissue sarcomas arising in the ET, but not in VR locations. In the latter histological grade and resection margins are the most important prognostic factors.

Keywords: Angiogenesis, Sarcoma, Extremity, Trunk, FGF, PDGF, VEGF, Visceral, Retroperitoneal

\section{Background}

Soft tissue sarcomas (STS) constitute a highly heterogeneous collection of tumors comprising over 50 histological subtypes, arising from mesenchymal tissue and capable of forming tumors in all parts of the human body [1]. This group amounts to $0.5-1 \%$ of the annual tumor burden with a mortality of about $40-60 \%$, resulting in an estimated 11280 cases and 3900 deaths in the US in 2012 [2].

\footnotetext{
*Correspondence: kilvaer@gmail.com

'Department of Oncology, University Hospital of North Norway, 9037 Tromso, Norway

Full list of author information is available at the end of the article
}

It is good practice to distinguish between STSs arising in the extremity \& trunk (ET), head \& neck (HN) and visceral \& retroperitoneal (VR) localizations as treatment and prognosis vary widely according to localization [3]. Further subdivision, according to histological type, malignancy grade, stage and vascular invasion among others, can be conducted [3]. Definitive treatment is radical surgery followed by radiotherapy in case of non-radical surgical margins [4]. Adjuvant chemotherapy for adult STS is still under investigation, and hence the routine use of such treatment is today limited to the palliative setting [5].

C Biomed Central

(c) 2014 Kilvaer et al.; licensee BioMed Central Ltd. This is an Open Access article distributed under the terms of the Creative Commons Attribution License (http://creativecommons.org/licenses/by/2.0), which permits unrestricted use, distribution, and reproduction in any medium, provided the original work is properly cited. The Creative Commons Public Domain Dedication waiver (http://creativecommons.org/publicdomain/zero/1.0/) applies to the data made available in this article, unless otherwise
stated. 
Angiogenesis is the process of forming new blood vessels from pre-existing ones. Folkman and coworkers proved this to be a pivotal step in carcinogenesis by showing that tumors would not grow beyond $>2 \mathrm{~mm}$ in diameter without forming vasculature [6,7]. In 2001, Hanahan and Weinberg, suggested angiogenesis as one of the hallmarks of cancer [8] and in the 2011 updated version angiogenesis was still considered one of the most important aspects of cancer progression [9].

Vascular endothelial growth factors (VEGF) and receptors (VEGFR) are pivotal in endothelial cell proliferation and sprouting during angio- and lymphangiogenesis [10]. Platelet-derived growth factors (PDGF) and receptors (PDGFR) play an important part in the regulation of tumor stroma through the recruitment of pericytes and vascular smooth muscle cells helping to stabilize newly formed vessels and through stimulation of stromal cells to produce VEGF-A and thus drive angiogenesis [11,12]. Fibroblast growth factors (FGF) and receptors (FGFR) drives endothelial cell proliferation and sprouting and activate several molecules involved in extracellular matrix remodelling including matrix metallo-proteinases and urokinase-like plasminogen activator [13].

Our group has previously reported on the expression of VEGF, PDGF and FGF families of growth factors in STSs of all sites [14-16]. This report investigates the differential impact of these growth factors in STSs arising in ET versus VR localizations.

\section{Methods}

\section{Patients and clinical samples}

Primary tumor tissue from anonymized patients diagnosed with STS at the University Hospital of North-Norway and the Hospitals of Arkhangelsk County, Russia, from 1973 through 2006, were collected. In total 496 patients were registered from the hospital databases. Of these, 388 patients were excluded from the study because of: missing clinical data $(n=86)$, inadequate formalin-fixed paraffin-embedded (FFPE) tissue blocks $(n=161)$, no surgery performed and/or metastasis present at the time of diagnosis $(n=55)$ or head and neck sarcomas ( $\mathrm{n}=13$ ). Thus 115 patients with STSs of the extremities and trunk wall and 66 patients with STSs of visceral or retroperitoneal origin, with complete medical records and FFPE tissue blocks were eligible.

This report includes follow-up data as of September 2009. The median follow-up was 53.9 (range 0.5-391.7) months for extremity and trunk patients and 59.4 (range 0.10-366.7) months for visceral and retroperitoneal patients. Complete demographic and clinical data were collected retrospectively. Formalin-fixed and paraffin-embedded tumor specimens were obtained from the archives of the Departments of Pathology at the University Hospital of North-Norway and the Hospitals of
Arkhangelsk County, Russia. The tumors were graded according to the French Fédération Nationale des centres de Lutte Contre le Cancer (FNCLCC) system and histologically subtyped according to the World Health Organization guidelines [1,17]. Wide resection margins were defined as wide local resection with free microscopic margins or amputation of the affected limb or organ.

\section{Microarray construction}

All sarcomas were histologically reviewed by two trained pathologists (S. Sorbye and A. Valkov) and the most representative areas of tumor cells (neoplastic mesenchymal cells) were carefully selected and marked on the hematoxylin and eosin $(\mathrm{H} / \mathrm{E})$ slide and sampled for the tissue microarray (TMA) blocks. The TMAs were assembled using a tissue-arraying instrument (Beecher Instruments, Silver Springs, MD). The Detailed methodology has been previously reported [18]. Briefly, we used a $0.6 \mathrm{~mm}$ diameter stylet, and the study specimens were routinely sampled with four replicate core samples from different areas of neoplastic tissue. Normal tissue from the patients was used as staining control.

To include all core samples, 12 TMA blocks were constructed. Multiple 5- $\mathrm{mm}$ sections were cut with a Micron microtome (HM355S) and stained by specific antibodies for immunohistochemistry (IHC) analysis.

\section{Immunohistochemistry}

The applied antibodies were subjected to in-house validation by the manufacturer for IHC analysis on paraffin-embedded material. The detailed methodology has previously been reported [14-16].

\section{Scoring of immunohistochemistry}

The ARIOL imaging system (Genetix, San Jose, CA) was used to scan the slides of antibody staining of the TMAs and the dominant staining intensity was scored as: 0 =negative; 1 = weak; 2 = intermediate; 3 = strong semi-qantitively on computer screen. The detailed methodology has previously been reported and cut-off values chosen were the same as in our previous studies [14-16]. High expression in tumor cells were defined as $\geq 1$ (VEGF-C), $\geq 1.5$ (PDGF-A, PDGF-C, PDGF-B, VEGF-A, VEGF-D, VEGFR-1-2 and -3 ) and $\geq 2$ (PDGF-D, PDGFR- $\alpha$, PDGFR- $\beta$, FGF2 and FGFR-1).

\section{Statistical methods}

All statistical analyses were done using the statistical package SPSS (Chicago, IL), version 16. The IHC scores from each observer were compared for interobserver reliability by use of a two-way random effect model with absolute agreement definition. The intraclass correlation coefficient (reliability coefficient) was obtained from these results. The Chi-square test and Fishers Exact test were 
Table 1 Prognostic clinicopathological variables as predictors for disease-specific survival, metastasis and local recurrence in patients with resected Extremitiy \& Trunk and Visceral \& Retroperitoneal soft-tissue sarcomas (univariate analyses, log rank test, $\mathrm{n}=115$ and 66 respectively)

\begin{tabular}{|c|c|c|c|c|c|c|c|c|c|c|c|c|c|c|}
\hline \multirow[b]{3}{*}{ Characteristics } & \multicolumn{7}{|c|}{ Extremity \& trunk } & \multicolumn{7}{|c|}{ Visceral \& retroperitoneal } \\
\hline & \multirow[b]{2}{*}{ Patients (n) } & \multicolumn{2}{|c|}{$\begin{array}{l}\text { Disease-specific } \\
\text { survival }\end{array}$} & \multicolumn{2}{|c|}{$\begin{array}{l}\text { Metastasis-free } \\
\text { survival }\end{array}$} & \multicolumn{2}{|c|}{$\begin{array}{l}\text { Recurrence-free } \\
\text { survival }\end{array}$} & \multirow[b]{2}{*}{ Patients (n) } & \multicolumn{2}{|c|}{$\begin{array}{l}\text { Disease-specific } \\
\text { survival }\end{array}$} & \multicolumn{2}{|c|}{$\begin{array}{l}\text { Metastasis-free } \\
\text { survival }\end{array}$} & \multicolumn{2}{|c|}{$\begin{array}{l}\text { Recurrence-free } \\
\text { survival }\end{array}$} \\
\hline & & $\begin{array}{l}5 \text {-Year } \\
\text { survival (\%) }\end{array}$ & $\mathbf{P}$ & $\begin{array}{l}5 \text {-Year } \\
\text { survival (\%) }\end{array}$ & $\mathbf{P}$ & $\begin{array}{l}5 \text {-Year } \\
\text { survival (\%) }\end{array}$ & $P$ & & $\begin{array}{l}5 \text {-Year } \\
\text { survival (\%) }\end{array}$ & $P$ & $\begin{array}{l}5 \text {-Year } \\
\text { survival (\%) }\end{array}$ & $\mathbf{P}$ & $\begin{array}{l}5 \text {-Year } \\
\text { survival (\%) }\end{array}$ & $\mathbf{P}$ \\
\hline \multicolumn{15}{|l|}{ Age } \\
\hline$\leq 20$ years & 12 & 42 & 0.431 & 42 & 0.129 & 73 & 0.690 & 1 & 0 & $<0.001$ & 100 & 0.112 & 100 & 0.786 \\
\hline $21-60$ years & 48 & 61 & & 63 & & 69 & & 36 & 71 & & 67 & & 71 & \\
\hline$>60$ years & 55 & 55 & & 69 & & 64 & & 29 & 43 & & 44 & & 62 & \\
\hline \multicolumn{15}{|l|}{ Gender } \\
\hline Male & 57 & 56 & 0.298 & 66 & 0.368 & 66 & 0.759 & 15 & 79 & 0.039 & 86 & 0.022 & 66 & 0.799 \\
\hline Female & 58 & 54 & & 61 & & 67 & & 51 & 51 & & 48 & & 68 & \\
\hline \multicolumn{15}{|l|}{ Patient nationality } \\
\hline Norwegian & 67 & 65 & 0.004 & 74 & 0.008 & 71 & 0.249 & 54 & 62 & 0.051 & 59 & 0.122 & 66 & 0.892 \\
\hline Russian & 48 & 42 & & 48 & & 57 & & 12 & 36 & & 46 & & 59 & \\
\hline \multicolumn{15}{|l|}{ Histological entity } \\
\hline Pleomorphic sarcoma & 48 & 42 & 0.004 & 61 & 0.001 & 56 & 0.664 & 6 & 50 & 0.917 & 67 & 0.264 & 40 & 0.274 \\
\hline Leiomyosarcoma & 9 & 100 & & 78 & & 78 & & 39 & 55 & & 46 & & 77 & \\
\hline Liposarcoma & 18 & 83 & & 94 & & 89 & & 13 & 62 & & 81 & & 54 & \\
\hline Fibrosarcoma & 12 & 57 & & 67 & & 55 & & 0 & & & & & & \\
\hline Angiosarcoma & 5 & 40 & & 20 & & 67 & & 2 & 50 & & 50 & & 50 & \\
\hline Rhabdomyosarcoma & 5 & 60 & & 60 & & 60 & & 1 & & & 100 & & 100 & \\
\hline MPNST & 5 & 53 & & 60 & & 60 & & 4 & 67 & & 100 & & 100 & \\
\hline Synovial sarcoma & 10 & 13 & & 30 & & 64 & & 1 & 100 & & 0 & & 0 & \\
\hline Sarcoma NOS & 3 & 100 & & 67 & & 67 & & 0 & & & & & & \\
\hline \multicolumn{15}{|l|}{ Tumor size } \\
\hline$<5 \mathrm{~cm}$ & 38 & 70 & 0.048 & 81 & 0.053 & 74 & 0.085 & 11 & 82 & 0.107 & 73 & 0.259 & 89 & 0.006 \\
\hline $5-10 \mathrm{~cm}$ & 45 & 48 & & 53 & & 61 & & 24 & 62 & & 62 & & 70 & \\
\hline$>10 \mathrm{~cm}$ & 30 & 49 & & 58 & & 62 & & 31 & 45 & & 45 & & 49 & \\
\hline Missing & 2 & & & & & & & & & & & & & \\
\hline
\end{tabular}


Table 1 Prognostic clinicopathological variables as predictors for disease-specific survival, metastasis and local recurrence in patients with resected Extremitiy \& Trunk and Visceral \& Retroperitoneal soft-tissue sarcomas (univariate analyses, log rank test, $\mathbf{n}=115$ and 66 respectively) (Continued)

\begin{tabular}{|c|c|c|c|c|c|c|c|c|c|c|c|c|c|c|}
\hline \multicolumn{15}{|c|}{ Malignancy grade } \\
\hline 1 & 29 & 89 & $<0.001$ & 89 & 0.001 & 85 & 0.054 & 23 & 78 & 0.005 & 77 & 0.051 & 86 & 0.046 \\
\hline 2 & 41 & 56 & & 65 & & 60 & & 29 & 46 & & 42 & & 58 & \\
\hline 3 & 45 & 35 & & 43 & & 57 & & 14 & 46 & & 47 & & 49 & \\
\hline \multicolumn{15}{|c|}{ Vascular invasion } \\
\hline Absent & 64 & 70 & $<0.001$ & 85 & $<0.001$ & 79 & $<0.001$ & 43 & 59 & 0.656 & 60 & 0.847 & 66 & 0.675 \\
\hline Present & 50 & 35 & & 33 & & 43 & & 20 & 51 & & 54 & & 78 & \\
\hline Missing & 1 & & & & & & & 3 & & & & & & \\
\hline \multicolumn{15}{|c|}{ Tumor depth } \\
\hline Superficial & 12 & 91 & 0.010 & 100 & 0.012 & 91 & 0.041 & 32 & & & & & & \\
\hline Deep & 103 & 51 & & 59 & & 63 & & 34 & & & & & & \\
\hline \multicolumn{15}{|c|}{ Resection margins } \\
\hline Wide & 61 & 66 & 0.004 & 72 & 0.045 & 82 & $<0.001$ & 50 & 65 & 0.021 & 59 & 0.654 & 90 & $<0.001$ \\
\hline Non-wide & 54 & 44 & & 54 & & 46 & & 16 & 50 & & 53 & & 44 & \\
\hline
\end{tabular}

Abbreviations: MPNST Malingnant peripheral nerve sheat tumor, NOS Not otherwise specified. 
Table 2 Angiogenic markers as predictors for disease-specific survival, metastasis and local recurrence in patients with resected soft-tissue sarcomas of the extremities or trunk (univariate analyses, log rank test, $n=115$ )

\begin{tabular}{|c|c|c|c|c|c|c|c|}
\hline \multirow[b]{2}{*}{ Marker expression } & \multirow[b]{2}{*}{ Patients (n) } & \multicolumn{2}{|c|}{ Disase-specific survival } & \multicolumn{2}{|c|}{ Metastasis-free survival } & \multicolumn{2}{|c|}{ Recurrence-free survival } \\
\hline & & 5-Year survival (\%) & $P$ & 5-Year survival (\%) & $\mathbf{P}$ & 5-Year survival (\%) & $\mathbf{P}$ \\
\hline \multicolumn{8}{|l|}{ PDGF-A } \\
\hline Low & 54 & 60 & 0.035 & 74 & 0.007 & 79 & 0.012 \\
\hline High & 58 & 52 & & 51 & & 55 & \\
\hline Missing & 3 & & & & & & \\
\hline \multicolumn{8}{|l|}{ PDGF-B } \\
\hline Low & 44 & 68 & 0.006 & 78 & 0.003 & 82 & 0.015 \\
\hline High & 68 & 48 & & 52 & & 56 & \\
\hline Missing & 3 & & & & & & \\
\hline \multicolumn{8}{|l|}{ PDGF-C } \\
\hline Low & 31 & 71 & 0.032 & 68 & 0.214 & 68 & 0.564 \\
\hline High & 80 & 50 & & 61 & & 65 & \\
\hline Missing & 4 & & & & & & \\
\hline \multicolumn{8}{|l|}{ PDGF-D } \\
\hline Low & 73 & 67 & 0.003 & 70 & 0.051 & 77 & 0.002 \\
\hline High & 40 & 34 & & 49 & & 42 & \\
\hline Missing & 2 & & & & & & \\
\hline \multicolumn{8}{|l|}{ PDGFR- $a$} \\
\hline Low & 69 & 67 & 0.002 & 74 & 0.002 & 77 & 0.011 \\
\hline High & 43 & 38 & & 42 & & 45 & \\
\hline Missing & 3 & & & & & & \\
\hline \multicolumn{8}{|l|}{ PDGFR- $\beta$} \\
\hline Low & 85 & 64 & 0.029 & 72 & 0.002 & 69 & 0.825 \\
\hline High & 24 & 32 & & 35 & & 58 & \\
\hline Missing & 6 & & & & & & \\
\hline \multicolumn{8}{|l|}{ VEGF-A } \\
\hline Low & 60 & 65 & 0.001 & 75 & 0.001 & 77 & 0.002 \\
\hline High & 51 & 43 & & 48 & & 51 & \\
\hline Missing & 4 & & & & & & \\
\hline \multicolumn{8}{|l|}{ VEGF-C } \\
\hline Low & 69 & 55 & 0.476 & 68 & 0.083 & 68 & 0.232 \\
\hline High & 38 & 60 & & 56 & & 63 & \\
\hline Missing & 8 & & & & & & \\
\hline \multicolumn{8}{|l|}{ VEGF-D } \\
\hline Low & 84 & 57 & 0.131 & 67 & 0.081 & 70 & 0.177 \\
\hline High & 29 & 50 & & 51 & & 55 & \\
\hline Missing & 2 & & & & & & \\
\hline \multicolumn{8}{|l|}{ VEGFR-1 } \\
\hline Low & 67 & 63 & 0.002 & 77 & $<0.001$ & 72 & 0.036 \\
\hline High & 44 & 46 & & 43 & & 58 & \\
\hline
\end{tabular}

Missing 
Table 2 Angiogenic markers as predictors for disease-specific survival, metastasis and local recurrence in patients with resected soft-tissue sarcomas of the extremities or trunk (univariate analyses, log rank test, $\mathbf{n}=115$ ) (Continued)

\begin{tabular}{|c|c|c|c|c|c|c|c|}
\hline \multicolumn{8}{|l|}{ VEGFR-2 } \\
\hline Low & 78 & 58 & 0.332 & 67 & 0.189 & 71 & 0.240 \\
\hline High & 28 & 52 & & 53 & & 57 & \\
\hline Missing & 9 & & & & & & \\
\hline \multicolumn{8}{|l|}{ VEGFR-3 } \\
\hline Low & 75 & 60 & 0.053 & 70 & 0.008 & 70 & 0.159 \\
\hline High & 34 & 45 & & 46 & & 57 & \\
\hline Missing & 6 & & & & & & \\
\hline \multicolumn{8}{|l|}{ FGF2 } \\
\hline Low & 75 & 61 & 0.033 & 66 & 0.214 & 70 & 0.648 \\
\hline High & 35 & 49 & & 56 & & 67 & \\
\hline Missing & 6 & & & & & & \\
\hline \multicolumn{8}{|l|}{ FGFR-1 } \\
\hline Low & 83 & 58 & 0.460 & 64 & 0.411 & 66 & 0.768 \\
\hline High & 26 & 47 & & 55 & & 71 & \\
\hline Missing & 6 & & & & & & \\
\hline
\end{tabular}

Abbreviations: PDGF Platelet-derived growth factor, PDGFR Platelet-derived growth factor receptor, VEGF Vascular endothelial growth factor, VEGFR Vascular endothelial growth factor receptor, FGF Fibroblast growth factor, FGFR Fibroblast growth factor receptor.

used to examine the association between molecular marker expression and various clinicopathological parameters. Univariate analyses were done using the Kaplan-Meier method, and statistical significance between survival curves was assessed by the log-rank test. Disease-specific survival (DSS) was determined from the date of diagnosis to the time of cancer related death. Metastasis-free survival (MFS) was defined from the date of diagnosis to the clinical appearance of the first metastasis. Recurrence-free survival (RFS), was defined from the date of diagnosis to the clinical appearance of the first recurrence. To assess the independent value of different pretreatment variables on survival, metastasis and local recurrence, in the presence of other variables, multivariate analyses were carried out using the Cox proportional hazards model. Only variables of significant value from the univariate analyses were entered into the Cox regression analysis. Probability for stepwise entry and removal was set at .05 and .10, respectively. The significance level used for all statistical tests was $\mathrm{P}<0.05$.

\section{Ethical clearance}

The Norwegian National Data Inspection Board and The Regional Committee for Research Ethics (Northern Norway) approved the study.

\section{Results}

\section{Clinicopathological variables}

The clinicopathological variables are summarized in Table 1. In the ET group, comprising 115 patients, median age was 59 (range 0-89) years, 50\% of the patients were male, 67 patients were Norwegian and 48 Russian and $68 \%$ of the tumors were located in the extremities. Of the histological subtypes represented, 48 were undifferentiated pleomorphic sarcomas, 18 liposarcomas, 12 fibrosarcomas, 10 synovial sarcomas, 9 leiomyosarcomas, 5 angiosarcomas, 5 rhabdomyosarcomas, 5 malignant peripheral nerve sheath tumors (MPNST) and 3 sarcoma not otherwise specified (NOS).

In the VR group, median age was 58 (range 13-88) years, $23 \%$ of the patients were male and 54 patients were Norwegian and 12 Russian. Of the histological subtypes represented, 39 were leiomyosarcomas, 13 liposarcomas, 6 pleomorphic sarcomas, 4 neurofibrosarcomas/ MPNSTs, 2 angiosarcomas, 1 rhabdomyosarcoma and 1 synovial sarcoma.

\section{Interobserver variability}

Interobserver scoring agreement was tested for PDGF-B, PDGFR- $\alpha$, VEGF-C, VEGFR-3, FGF2 and FGFR1 and found to be good (0.77-0.90, $\mathrm{P}<0.001)$ [14-16].

\section{Univariate analyses}

The impact of the clinicopathological variables on DSS, MFS and RFS in the ET group are summarized in Table 1. Patient nationality $(P=0.004)$, histological entity $(\mathrm{p}=0.004)$, tumor size $(\mathrm{p}=0.048)$, malignancy grade $(\mathrm{P}<0.001)$, vascular invasion $(\mathrm{P}<0.001)$, tumor depth $(\mathrm{P}=0.010)$ and resection margins $(\mathrm{P}=0.004)$ were all prognostic indicators of DSS. Patient nationality $(\mathrm{P}=0.008)$, 
Table 3 Angiogenic markers as predictors for disease-specific survival, metastasis and local recurrence in patients with resected visceral \& retroperitoneal soft-tissue sarcomas (univariate analyses, log rank test, $n=66$ )

\begin{tabular}{|c|c|c|c|c|c|c|c|}
\hline \multirow[b]{2}{*}{ Marker expression } & \multirow[b]{2}{*}{ Patients (n) } & \multicolumn{2}{|c|}{ Disase-specific survival } & \multicolumn{2}{|c|}{ Metastasis-free survival } & \multicolumn{2}{|c|}{ Recurrence-free survival } \\
\hline & & 5-Year survival (\%) & $\mathbf{P}$ & 5-Year survival (\%) & $\mathbf{P}$ & 5-Year survival (\%) & $\mathbf{P}$ \\
\hline \multicolumn{8}{|l|}{ PDGF-A } \\
\hline Low & 23 & 49 & 0.473 & 63 & 0.593 & 65 & 0.315 \\
\hline High & 39 & 63 & & 54 & & 73 & \\
\hline Missing & 4 & & & & & & \\
\hline \multicolumn{8}{|l|}{ PDGF-B } \\
\hline Low & 14 & 54 & 0.604 & 82 & 0.088 & 61 & 0.291 \\
\hline High & 48 & 59 & & 51 & & 73 & \\
\hline Missing & 4 & & & & & & \\
\hline \multicolumn{8}{|l|}{ PDGF-C } \\
\hline Low & 20 & 39 & 0.297 & 59 & 0.986 & 53 & 0.045 \\
\hline High & 41 & 65 & & 56 & & 76 & \\
\hline Missing & 5 & & & & & & \\
\hline \multicolumn{8}{|l|}{ PDGF-D } \\
\hline Low & 48 & 52 & 0.078 & 52 & 0.197 & 70 & 0.343 \\
\hline High & 15 & 80 & & 73 & & 59 & \\
\hline Missing & 3 & & & & & & \\
\hline \multicolumn{8}{|l|}{ PDGFR- $a$} \\
\hline Low & 41 & 56 & 0.672 & 61 & 0.527 & 71 & 0.761 \\
\hline High & 21 & 61 & & 51 & & 66 & \\
\hline Missing & 4 & & & & & & \\
\hline \multicolumn{8}{|l|}{ PDGFR- $\beta$} \\
\hline Low & 58 & 57 & 0.360 & 54 & 0.532 & 70 & 0.766 \\
\hline High & 4 & 75 & & 75 & & 75 & \\
\hline Missing & 4 & & & & & & \\
\hline \multicolumn{8}{|l|}{ VEGF-A } \\
\hline Low & 34 & 51 & 0.326 & 64 & 0.719 & 64 & 0.054 \\
\hline High & 29 & 68 & & 53 & & 79 & \\
\hline Missing & 3 & & & & & & \\
\hline \multicolumn{8}{|l|}{ VEGF-C } \\
\hline Low & 34 & 66 & 0.402 & 69 & 0.071 & 62 & 0.051 \\
\hline High & 29 & 50 & & 45 & & 84 & \\
\hline Missing & 3 & & & & & & \\
\hline \multicolumn{8}{|l|}{ VEGF-D } \\
\hline Low & 34 & 60 & 0.856 & 62 & 0.388 & 63 & 0.116 \\
\hline High & 30 & 55 & & 51 & & 78 & \\
\hline Missing & 2 & & & & & & \\
\hline \multicolumn{8}{|l|}{ VEGFR-1 } \\
\hline Low & 37 & 55 & 0.724 & 63 & 0.358 & 70 & 0.510 \\
\hline High & 25 & 63 & & 49 & & 71 & \\
\hline
\end{tabular}


Table 3 Angiogenic markers as predictors for disease-specific survival, metastasis and local recurrence in patients with resected visceral \& retroperitoneal soft-tissue sarcomas (univariate analyses, log rank test, $\mathbf{n}=66$ ) (Continued)

\begin{tabular}{|c|c|c|c|c|c|c|c|}
\hline \multicolumn{8}{|l|}{ VEGFR-2 } \\
\hline Low & 44 & 55 & 0.858 & 63 & 0.446 & 69 & 0.821 \\
\hline High & 19 & 67 & & 48 & & 75 & \\
\hline Missing & 3 & & & & & & \\
\hline \multicolumn{8}{|l|}{ VEGFR-3 } \\
\hline Low & 36 & 54 & 0.552 & 59 & 0.821 & 65 & 0.220 \\
\hline High & 25 & 61 & & 52 & & 76 & \\
\hline Missing & 5 & & & & & & \\
\hline \multicolumn{8}{|l|}{ FGF2 } \\
\hline Low & 39 & 56 & 0.805 & 51 & 0.214 & 74 & 0.748 \\
\hline High & 20 & 65 & & 67 & & 66 & \\
\hline Missing & 7 & & & & & & \\
\hline \multicolumn{8}{|l|}{ FGFR-1 } \\
\hline Low & 43 & 45 & 0.023 & 56 & 0.385 & 68 & 0.448 \\
\hline High & 20 & 89 & & 63 & & 78 & \\
\hline Missing & 3 & & & & & & \\
\hline
\end{tabular}

Abbreviations: PDGF Platelet-derived growth factor, PDGFR Platelet-derived growth factor receptor, VEGF Vascular endothelial growth factor, VEGFR Vascular endothelial growth factor receptor, FGF Fibroblast growth factor, FGFR Fibroblast growth factor receptor.

histological entity $(P=0.001)$, malignancy grade $(P=0.001)$, vascular invasion $(\mathrm{P}<0.001)$, tumor depth $(\mathrm{P}=0.012)$ and resection margins $(\mathrm{P}=0.045)$ were prognostic indicators of MFS. Finally, vascular invasion $(\mathrm{P}<0.001)$, tumor depth $(P=0.041)$ and resection margins $(P<0.001)$ were prognostic indicators of RFS.

The impact of the angiogenic markers on DSS, MFS and RFS in the ET group are summarized in Table 2. PDGF-A $(\mathrm{P}=0.035)$, PDGF-B $(\mathrm{P}=0.006)$, PDGF-C $(\mathrm{P}=0.032), \quad$ PDGF-D $(\mathrm{P}=0.003), \quad$ PDGFR $-\alpha$ $(\mathrm{P}=0.002)$, PDGFR- $\beta(\mathrm{P}=0.029)$, VEGF-A $(\mathrm{P}=0.001)$, VEGFR-1 $(\mathrm{P}=0.001)$ and FGF2 $(\mathrm{P}=0.033)$ were prognostic indicators of DSS. PDGF-A ( $\mathrm{P}=0.007)$, PDGF-B $(\mathrm{P}=0.003)$, PDGFR- $\alpha \quad(P=0.002), \quad$ PDGFR- $\beta \quad(P=0.002)$, VEGF-A $(\mathrm{P}=0.001)$, VEGFR-1 $(\mathrm{P}<0.001)$ and VEGFR-3 $(\mathrm{P}=0.008)$ were prognostic indicators of MFS. PDGF-A $(\mathrm{P}=0.012)$, PDGF-B $(P=0.015), \quad$ PDGFR- $\alpha \quad(P=0.011)$, VEGF-A $(\mathrm{P}=0.002)$ and VEGFR-1 $(\mathrm{P}=0.036)$ were prognostic indicators of RFS.

The impact of the clinicopathological variables on DSS, MFS and RFS in the VR group are summarized in Table 1. Age $(P<0.001)$, gender $(P=0.039)$, malignancy grade $(P=0.005)$ and resection margins $(P=0.021)$ were prognostic indicators of DSS. Gender $(\mathrm{P}=0.022)$ was a prognostic indicator of MFS and tumor size $(\mathrm{P}=0.006)$, malignancy grade $(\mathrm{P}=0.046)$ and resection margins $(\mathrm{P}<0.001)$ were prognostic indicators of RFS.

The impact of angiogenic markers on DSS, MFS and RFS in the VR group is summarized in Table 3.
FGRF-1 ( $\mathrm{P}=0.023)$ was the only prognostic indicator for DSS and PDGF-C $(\mathrm{P}=0.045)$ for RFS.

\section{Multivariate cox proportional hazards analysis}

Table 4 presents multivariate analyses of clinicopathological and angiogenic marker variables with respect to DSS, MFS and RFS in the ET and VR groups, respectively.

In the ET group, high malignancy grade $(\mathrm{P}<0.001)$, the presence of vascular invasion $(\mathrm{P}=0.011)$, non-wide resection margins $(\mathrm{P}=0.039)$ and high expression of PDGF-D $(\mathrm{HR}=1.863,95 \% \mathrm{CI}=1.057-3.283, \quad \mathrm{P}=0.031)$ were significant independent prognostic indicators of DSS. Further, the presence of vascular invasion $(\mathrm{P}<0.001)$ and high expression of VEGFR-1 $(\mathrm{HR}=2.106,95 \%$ $\mathrm{CI}=1.038-4.272, \mathrm{P}=0.039)$ were significant independent prognostic factors of MFS, while the presence of vascular invasion $(\mathrm{P}=0.045)$, non-wide resection margins $(\mathrm{P}=0.008)$ and high expression of VEGF-A (HR 2.095, 95\% CI $1.028-4.271, \mathrm{P}=0.042$ ) were significant independent prognostic factors of RFS.

In the VR group, high malignancy grade $(P=0.003)$ and non-wide resection margins $(\mathrm{P}=0.014)$ were significant independent adverse prognostic indicators of DSS whereas high FGFR-1 expression ( $\mathrm{HR}=0.243$, 95\% CI $=0.095-0.618, \quad \mathrm{P}=0.003$ ) was an independent positive prognostic indicator of DSS. Female gender $(\mathrm{P}=0.038)$ was an independent negative prognostic indicator of MFS while non-wide resection margins $(\mathrm{P}<0.001)$ was an independent negative prognostic indicator of RFS. 
Table 4 Multivariate analyses of clinopathological variables and angiogenic markers as prognostic values for disease-specific survival, metastasis and local recurrence in patients with resected soft-tissue sarcomas of the trunk or extremities (cox proportional hazards test)

\begin{tabular}{|c|c|c|c|c|c|c|c|c|c|}
\hline \multirow[b]{2}{*}{ Variable } & \multicolumn{2}{|c|}{ Disase-specific survival } & \multirow[b]{2}{*}{$\mathbf{P}$} & \multicolumn{2}{|c|}{ Metastasis-free survival } & \multirow[b]{2}{*}{$\mathbf{P}$} & \multicolumn{2}{|c|}{ Recurrence-free survival } & \multirow[b]{2}{*}{$\mathbf{P}$} \\
\hline & HR & $95 \% \mathrm{Cl}$ & & HR & $95 \% \mathrm{Cl}$ & & $\mathrm{HR}$ & $95 \% \mathrm{Cl}$ & \\
\hline \multicolumn{10}{|c|}{ Extremity \& trunk } \\
\hline \multicolumn{10}{|c|}{ Malignancy grade } \\
\hline 1 & 1.000 & & $<0.001^{*}$ & & & & & & \\
\hline 2 & 4.066 & $1.389-11.901$ & 0.010 & & & & & & \\
\hline 3 & 6.025 & $2.058-17.634$ & 0.001 & & & & & & \\
\hline \multicolumn{10}{|c|}{ Vascular invasion } \\
\hline Absent & 1.000 & & & 1.000 & & & 1.000 & & \\
\hline Present & 2.141 & $1.188-3.859$ & 0.011 & 5.284 & 2.418-11.544 & $<0.001$ & 2.135 & $1.019-4.475$ & 0.045 \\
\hline \multicolumn{10}{|c|}{ Resection margins } \\
\hline Wide & 1.000 & & & & & & 1.000 & & \\
\hline Non-wide & 1.818 & $1.032-3.203$ & 0.039 & & & & 2.687 & $1.289-5.602$ & 0.008 \\
\hline \multicolumn{10}{|l|}{ PDGF-B } \\
\hline Low & & & & & & & 1.000 & & \\
\hline High & & & & & & & 2.099 & $0.937-4.706$ & 0.072 \\
\hline \multicolumn{10}{|l|}{ PDGF-D } \\
\hline Low & 1.000 & & & & & & 1.000 & & \\
\hline High & 1.863 & $1.057-3.283$ & 0.031 & & & & 1.844 & $0.931-3.653$ & 0.079 \\
\hline \multicolumn{10}{|l|}{ VEGF-A } \\
\hline Low & & & & & & & 1.000 & & \\
\hline High & & & & & & & 2.095 & $1.028-4.271$ & 0.042 \\
\hline
\end{tabular}

VEGFR-1

\section{Low}

High

Visceral \& retroperitoneal

\section{Gender}

Male

Female

Malignancy grade

$\begin{array}{llll}1 & 1.000 & & 0.003^{*} \\ 2 & 4.812 & 1.823-12.705 & 0.002 \\ 3 & 5.646 & 1.790-17.804 & 0.003 \\ \begin{array}{l}\text { Resection margins } \\ \text { Wide }\end{array} & & \\ \text { Non-wide } & 1.000 & & \\ \text { PDGF-C } & 2.712 & 1.222-6.018 & 0.014 \\ \text { Low } & & & \\ \text { High } & & & \end{array}$

$0.003^{*}$

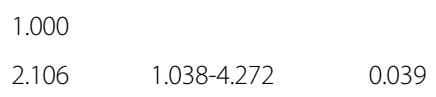

1.000

0.061

$\begin{array}{lll}2.069 & 0.630-6.794 & 0.231\end{array}$

$\begin{array}{lll}5.665 & 1.330-24.123 & 0.019\end{array}$

1.000

$11.996 \quad 3.128-46.005<0.001$

1.000

$\begin{array}{lll}0.413 & 0.157-1.089 & 0.074\end{array}$

FGFR-1
Low
1.000
High
0.243
0.095-0.618
0.003

Abbreviations: HR Hazard ratio, CI Confidence interval, VEGF Vascular endothelial growth factor, VEGFR Vascular endothelial growth factor receptor, PDGFR Platelet-derived growth factor receptor, FGFR Fibroblast growth factor receptor, *overall significance as prognostic factor. 


\section{Discussion and conclusions}

In our univariate analyses high expression of most examined angiogenic markers were prognosticators of DSS and/or MFS and/or RFS in the ET group. Further, PDGF-D was an independent negative prognostic indicator of DSS, VEGFR-1 an independent negative prognostic indicator of MFS and VEGF-A an independent negative prognostic indicator of RFS. In contrast, only FGFR-1 was a prognosticator of DSS in both the univariate and multivariate analyses of the VR group. To our knowledge, this is the first comparison of the expression of angiogenic molecules in ET versus VR STSs.

Current knowledge of the importance of tumor localization (ET versusVR tumors) when it comes to the prognostic impact of angiogenic markers in STSs is limited. Yudoh et. al. investigated the level of VEGF-A in tissue from ET patients and found high levels to predict survival, local recurrence and metastasis [18]. We have previously reported on the expression of PDGFs, VEGFs and FGFs in a larger cohort of STS of mixed sites and histology and found high expression of VEGFR-3, PDGF-B and FGF2 to have independent negative prognostic impact on DSS [14-16]. When comparing the expression of angiogenic markers based on tumor location, it becomes apparent that these variables almost exclusively have prognostic impact in STS arising in the ET group (Tables 2, 3 and 4). This difference could to some extent be due to a smaller number of patients in the VR group, with a resulting increased risk of false negative results. However, near all angiogenic markers showed significant prognostic impact in the univariate analyses of the ET group, whereas only FGFR-1 showed prognostic impact in the VR group. Table 1 summarizes the clinopathological values in the ET and VR groups and it is apparent that the VR group contains a higher percentage of leiomysarcomas and liposarcomas. The different distribution of histologies between the ET and VR groups might suggest that angiogenic markers have higher impact in STSs arising in ET locations. Another explanation may be that ET tumors, even the slow growing ones, will produce symptoms when they reach a certain size due to limits created by connective and muscle tissue and blood and lymph vessels. VR tumors could in contrast grow to significant size before producing symptoms. This may explain our results as VR tumors in many cases only are found after the angiogenic switch have occurred, thus the impact of angiogenic markers have been negated in these tumors.

In the PDGF-axis, all markers were prognosticators of DSS, all but PDGF-C were prognosticators of MFS and all but PDGF-C and PDGFR- $\beta$ were prognosticators of RFS in the ET group (Table 2), while none of the PDGFs were prognosticators in the VR group. Further, PDGF-D was found to be an independent negative prognostic factor for DSS in the ET group. In our previous study,
PDGF-B was an independent prognosticator of DSS [15], and in this study PDGF-D is an independent prognosticator of DSS. PDGF-B binds all PDGFRs while PDGF-D binds PDGFR- $\alpha \beta$ and- $\beta \beta$ [11]. Both PDGF-B and PDGF-D has been shown to exhibit similar and extensive angiogenic and transforming abilities [19,20]. Although our results cannot distinguish whether PDGF signalling drives tumor development through angiogenesis or other pathways, they strongly suggest PDGF signalling to be an important part of STS growth and progression.

In the VEGF-axis, VEGF-A, and VEGFR-1 were prognosticators of DSS, MFS and RFS in the ET group, while none of the VEGFs were prognosticators in the VR group (Table 2). Further, VEGFR-1 was an independent prognostic indicator of MFS and VEGF-A was an independent prognostic indicator of RFS in the ET group. VEGF-A signalling is the major angiogenic pathway, and high tumor expression and availability in serum has previously been associated with malignancy grade, metastasis, local recurrence and worse overall survival in STS patients [18,21-26]. VEGFR-1 is thought to modulate VEGF-A signalling through VEGFR-2, has anti-angiogenic properties in its soluble form, and has been linked to metastasis in experimental studies suggesting a feasible biological link for our finding in these STS patients $[27,28]$. This latter finding is quite interesting as antibodies and small-molecules targeting VEGFR-1 are being developed [29,30].

In the FGF-axis, FGF-2 was an unfavorable prognostic indicator of DSS in ET group. FGF2 is thought to drive cellcycling, activate extracellular matrix remodelling and to rescue PDGF-B and VEGF-A driven angiogenesis in the presence of their respective inhibitors [13,31,32]. Surprisingly, FGFR-1 was an independent positive indicator of DSS in the VR group. To our knowledge these are new data, but these results have to be validated before a firm conclusion may be drawn due to the low number of patients.

This study enhances our current knowledge on angiogenic prognosticators in STSs, strongly indicates the involvement of the PDGF and VEGF pathways in ET STS development and adds to the growing body of evidence suggesting that STSs of different sites and histology should be analyzed independently in future studies. Further emphasis should also be put on validating VEGFR-1 as a predictor of MFS in ET STS patients, as these patients may benefit from adjuvant therapy targeting VEGFR-1.

\section{Competing interests}

The authors declare that they have no competing interests.

\section{Authors' contributions}

All authors participated in designing the study, interpreting the results and in the writing of the paper. TK, AV, SWS and ES collected the clinical and demographic data. TK, SWS and AV scored the TMAs. TK and TD conducted the statistical analyses. TK drafted the manuscript. All authors read and approved the final manuscript. 


\section{Author details}

${ }^{1}$ Department of Oncology, University Hospital of North Norway, 9037 Tromso, Norway. ${ }^{2}$ Institute of Medical Biology, University of Tromso, Tromso, Norway. ${ }^{3}$ Department of Clinical Pathology, University Hospital of North Norway, Tromso, Norway. ${ }^{4}$ Institute of Clinical Medicine, University of Tromso, Tromso, Norway.

Received: 2 November 2013 Accepted: 16 January 2014

Published: 20 January 2014

\section{References}

1. Fletcher CDM, Unni KK, Mertens F: Pathology and genetics of tumours of soft tissue and bone. World Health Organization Classification of Tumours. Lyon: IARC Press; 2002:415

2. Siegel R, Naishadham D, Jemal A: Cancer statistics, 2012. CA Cancer J Clin 2012, 62(1):10-29.

3. Group E.E.S.N.W: Soft tissue and visceral sarcomas: ESMO Clinical Practice Guidelines for diagnosis, treatment and follow-up. Ann Oncol 2012, 23(Suppl 7):vii92-vii99.

4. Dickinson $I C$, et al: Surgical margin and its influence on survival in soft tissue sarcoma. ANZ J Surg 2006, 76(3):104-109.

5. Blay JY, Le Cesne A: Adjuvant chemotherapy in localized soft tissue sarcomas: still not proven. Oncologist 2009, 14(10):1013-1020.

6. Folkman J: Anti-angiogenesis: new concept for therapy of solid tumors. Ann Surg 1972, 175(3):409-416.

7. Folkman J, et al: Isolation of a tumor factor responsible for angiogenesis. J Exp Med 1971, 133(2):275-288.

8. Hanahan D, Weinberg RA: The hallmarks of cancer. Cell 2000, 100(1):57-70.

9. Hanahan D, Weinberg RA: Hallmarks of cancer: the next generation. Cell 2011, 144(5):646-674

10. Tammela $T$, et al: The biology of vascular endothelial growth factors Cardiovasc Res 2005, 65(3):550-563.

11. Fredriksson L, Li H, Eriksson U: The PDGF family: four gene products form five dimeric isoforms. Cytokine Growth Factor Rev 2004, 15(4):197-204

12. Ostman A: PDGF receptors-mediators of autocrine tumor growth and regulators of tumor vasculature and stroma. Cytokine Growth Factor Rev 2004, 15(4):275-286

13. Presta $M$, et al: Fibroblast growth factor/fibroblast growth factor receptor system in angiogenesis. Cytokine Growth Factor Rev 2005, 16(2):159-178.

14. Kilvaer TK, et al: Fibroblast growth factor 2 orchestrates angiogenic networking in non-GIST STS patients. J Trans/ Med 2011, 9:104.

15. Kilvaer TK, et al: Platelet-derived growth factors in non-GIST soft-tissue sarcomas identify a subgroup of patients with wide resection margins and poor disease-specific survival. Sarcoma 2010, 2010:751304.

16. Kilvaer TK, et al: Profiling of VEGFs and VEGFRs as prognostic factors in soft tissue sarcoma: VEGFR-3 is an independent predictor of poor prognosis. PLoS One 2010, 5(12):e15368.

17. Guillou $L$, et al: Comparative study of the National Cancer Institute and French Federation of Cancer Centers Sarcoma Group grading systems in a population of 410 adult patients with soft tissue sarcoma. J Clin Oncol 1997, 15(1):350-362.

18. Yudoh $\mathrm{K}$, et al: Concentration of vascular endothelial growth factor in the tumour tissue as a prognostic factor of soft tissue sarcomas. $\mathrm{Br} J$ Cancer 2001, 84(12):1610-1615.

19. Tallquist M, Kazlauskas A: PDGF signaling in cells and mice. Cytokine Growth Factor Rev 2004, 15(4):205-213.

20. $\mathrm{Li} \mathrm{H}$, et al: PDGF-D is a potent transforming and angiogenic growth factor. Oncogene 2003, 22(10):1501-1510.

21. Chao C, et al: Vascular endothelial growth factor and soft tissue sarcomas: tumor expression correlates with grade. Ann Surg Oncol 2001, 8(3):260-267.

22. Graeven U, et al: Serum levels of vascular endothelial growth factor and basic fibroblast growth factor in patients with soft-tissue sarcoma. J Cancer Res Clin Oncol 1999, 125(10):577-581.

23. Hayes AJ, et al: Serum vascular endothelial growth factor as a tumour marker in soft tissue sarcoma. Br J Surg 2004, 91(2):242-247.

24. Yoon SS, et al: Circulating angiogenic factor levels correlate with extent of disease and risk of recurrence in patients with soft tissue sarcoma. Ann Oncol 2004, 15(8):1261-1266.
25. Yoon SS, et al: Angiogenic profile of soft tissue sarcomas based on analysis of circulating factors and microarray gene expression. J Surg Res 2006, 135(2):282-290.

26. Adams $\mathrm{RH}$, Alitalo K: Molecular regulation of angiogenesis and lymphangiogenesis. Nat Rev Mol Cell Biol 2007, 8(6):464-478.

27. Hiratsuka $\mathrm{S}$, et al: MMP9 induction by vascular endothelial growth factor receptor-1 is involved in lung-specific metastasis. Cancer Cell 2002, 2(4):289-300.

28. Kaplan RN, et al: VEGFR1-positive haematopoietic bone marrow progenitors initiate the pre-metastatic niche. Nature 2005, 438(7069):820-827.

29. Lorusso PM, et al: Icrucumab, a fully human monoclonal antibody against the vascular endothelial growth factor receptor-1, in the treatment of patients with advanced solid malignancies: a Phase 1 study. Invest New Drugs 2013:1-9.

30. Hepgur $M$, et al: Tivozanib in the treatment of renal cell carcinoma. Biologics 2013, 7:139-148.

31. Welti JC, et al: Fibroblast growth factor 2 regulates endothelial cell sensitivity to sunitinib. Oncogene 2011, 30(10):1183-1193.

32. Ohshima $M$, et al: bFGF rescues imatinib/STI571-induced apoptosis of sis-NIH3T3 fibroblasts. Biochem Biophys Res Commun 2009, 381(2):165-170.

doi:10.1186/1472-6890-14-5

Cite this article as: Kilvaer et al:: The VEGF- and PDGF-family of angiogenic markers have prognostic impact in soft tissue sarcomas arising in the extremities and trunk. BMC Clinical Pathology 2014 14:5.

\section{Submit your next manuscript to BioMed Central and take full advantage of:}

- Convenient online submission

- Thorough peer review

- No space constraints or color figure charges

- Immediate publication on acceptance

- Inclusion in PubMed, CAS, Scopus and Google Scholar

- Research which is freely available for redistribution 